\title{
Isolated senile plaque cores in Alzheimer's disease and Down's syndrome show differences in morphology
}

\author{
DAVID ALLSOP,* MICHAEL KIDD, $\dagger$ MICHAEL LANDON,* \\ ANNETTE TOMLINSON $\dagger$ \\ From the Departments of Biochemistry* and Human Morphology, $\dagger$ University of Nottingham Medical School, \\ Queen's Medical Centre, Nottingham, UK
}

SUMMARY Frontal and temporal cortical tissue from the brains of elderly cases of Down's syndrome was used to make preparations of neuronal cell bodies containing senile plaque cores. Polarisation microscopy revealed normal "classical" plaque cores, and also a high proportion of unusual "amorphous" plaque cores which we have not seen in Alzheimer's disease. These two forms were easily distinguished by electron microscopy. This suggests that late Down's syndrome may not be an exact model for Alzheimer's disease.

Most individuals with Down's syndrome who survive to middle age ${ }^{12}$ develop the neuropathological characteristics of Alzheimer's disease, namely the presence of numerous senile plaques and neurofibrillary tangles in the cerebral cortex $;^{1-7}$ the degeneration of neurons in the nucleus basalis of Meynert; ${ }^{89}$ and an associated loss of cholinergic function. ${ }^{10}$ In Down's syndrome $\mathrm{s}^{5-7}$ the paired helical filaments forming the neurofibrillary tangles have the same dimensions and periodicity as those in Alzheimer's disease. ${ }^{11}$ The ultrastructure of the senile plaque in the latter condition has been well described, ${ }^{12-14}$ the typical mature plaque consisting of a central core of closely-packed, radiating amyloid fibrils, surrounded by a rim of degenerating neurites and synapses. Several reports $^{1-7}$ suggest that the plaques in Down's syndrome are similar to this in morphology. The plaque amyloid in both Down's syndrome and Alzheimer's disease $^{15}$ is formed from a c. $4,000 \mathrm{~mol}$ wt protein $(\beta$ protein), of unknown function, first isolated by Glenner and Wong ${ }^{1617}$ from cerebrovascular amyloid. It is likely that the paired helical filaments of neurofibrillary tangles are also a different form of this same protein, ${ }^{18}$ although this has not been established with certainty.

We have shown ${ }^{19} 20$ that some preparations of neuronal cell bodies produced from frozen post-mortem

Address for reprint requests: Dr D Allsop, Department of Biochemistry, University of Nottingham Medical School, Queen's Medical Centre, Nottingham NG7 2UH, UK.

Received 10 September 1985.

Accepted 16 October 1985
Alzheimer brains contain intact plaque cores lacking any of the surrounding rim. These isolated plaque cores are identical in size and appearance to those seen in situ, and the vast majority of them have a clearly-defined cross under the polarising microscope after staining with Congo red. In this report neuronal preparations were produced from the brains of four cases of Down's syndrome and one further case of Alzheimer's disease. Two of the Down's syndrome preparations were found to contain sufficient numbers of plaque cores for morphological study. In both cases polarisation microscopy revealed both normal plaque cores and a high proportion of unusual plaque cores with a strikingly different appearance. These unusual plaque cores were again not seen in the neuronal preparation from the Alzheimer's disease brain. In an electron microscopical study, the isolated plaque cores from one of the Down's syndrome brains yielded profiles which corresponded well to the light microscope appearance of the unusual plaque cores. Similar profiles were not found in the Alzheimer's disease brain which served as a control. This suggests that, contrary to previous indications, late Down's syndrome may not be an exact model for Alzheimer's disease, since these two conditions show some significant differences in plaque morphology.

Preliminary observations relating to this work have been reported previously. ${ }^{21}$

\section{Materials and methods}

Dr GP Reynolds kindly provided brain tissue from four cases of Down's syndrome (M3, M4, M5, M6) from the MRC Brain Tissue Bank, Addenbrookes Hospital, Cam- 

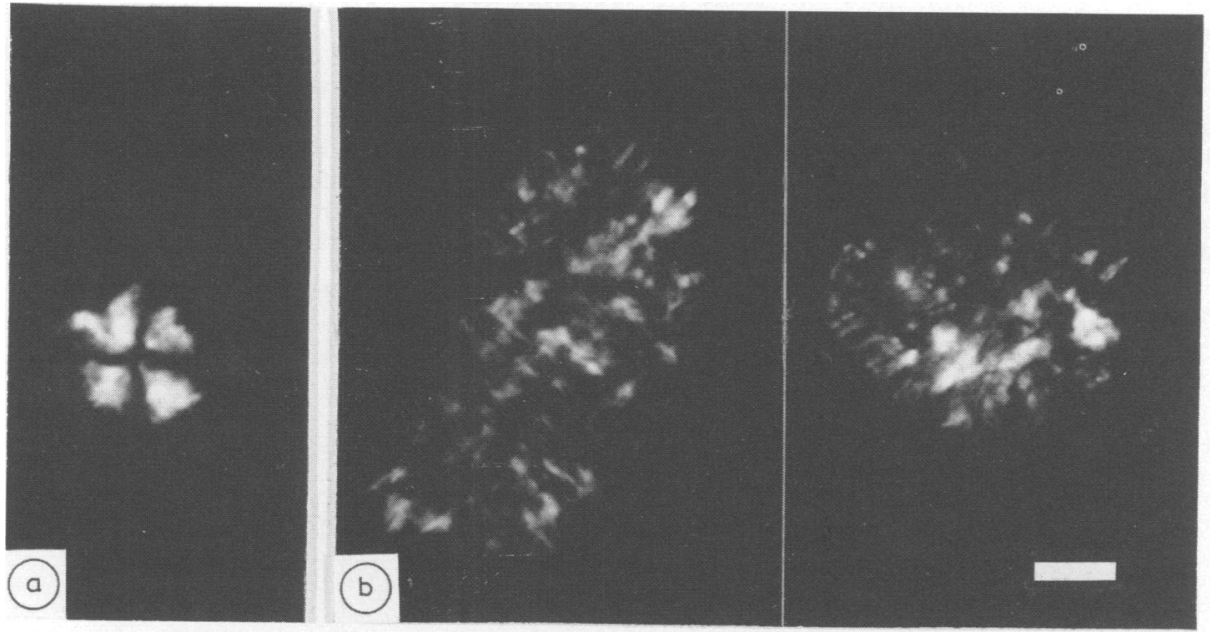

Fig 1 Senile plaque cores in the neuronal preparation from Down's syndrome case M3. The preparation was stained by Congo red and examined between crossed polarizers. (a) classical plaque core.

(b) Amorphous plaque cores. Bar $=10 \mu \mathrm{m}$.

(a)

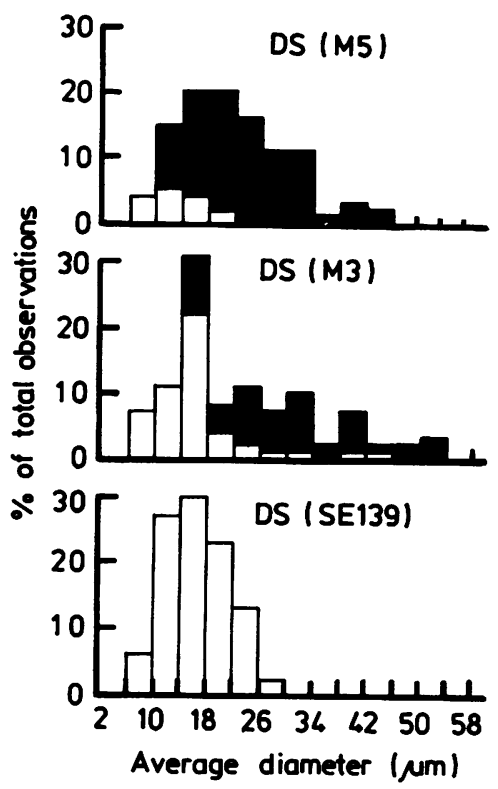

(b)

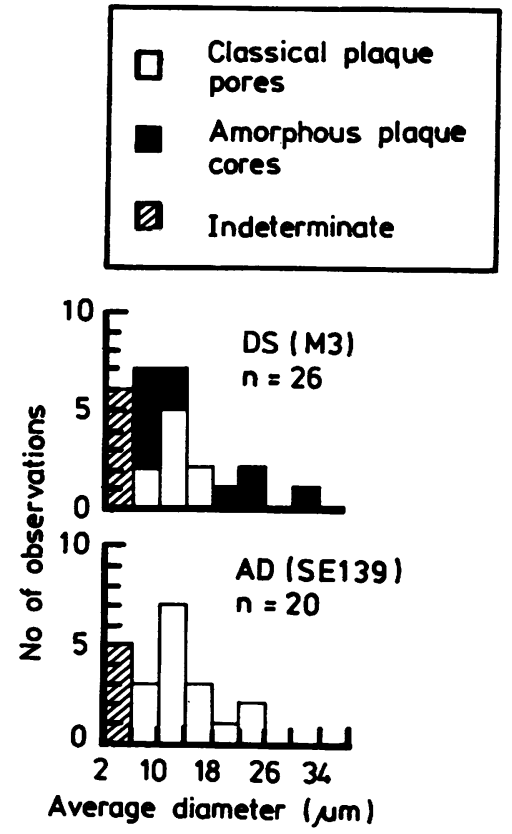

Fig 2 Numbers of classical and amorphous plaque cores in different size classes. (a) Light microscope observations on isolated plaque cores in neuronal preparations (in each case $n>100$ ). (b) Electron microscope observations on embedded and sectioned neuronal preparations. Note that it was impossible with profiles of diameter $<6 \mu \mathrm{m}$ to distinguish classical and amorphous forms. 
bridge. The sex and ages at death were M64; M,52; F,59; F,54 respectively. The Alzheimer's disease brain, case SE139 (F, age 81 ) was kindly provided by Dr IA Ansell of Nottingham City Hospital.

The enriched neuronal fractions were prepared from frontal or temporal cortex exactly as described previously. ${ }^{1920}$ Samples for light microscopy were suspended in $0.25 \%(\mathrm{w} / \mathrm{v})$ aq. Congo red and examined under a Vickers polarising microscope. For size measurements, the plaque cores were traced by means of a drawing tube attachment. Samples for electron microscopy were fixed in $3 \%$ glutaraldehyde, $0 \cdot 1 \mathrm{M}$ phosphate buffer $\mathrm{pH} 7.5$ for 3 hours at room temperature, post-fixed in $1 \%$ osmium tetroxide, dehydrated, and embedded in Araldite resin. Thin sections were cut on a Reichert Om U3 ultramicrotome, stained on the grid with ethanolic uranyl acetate followed by lead citrate, and exam-
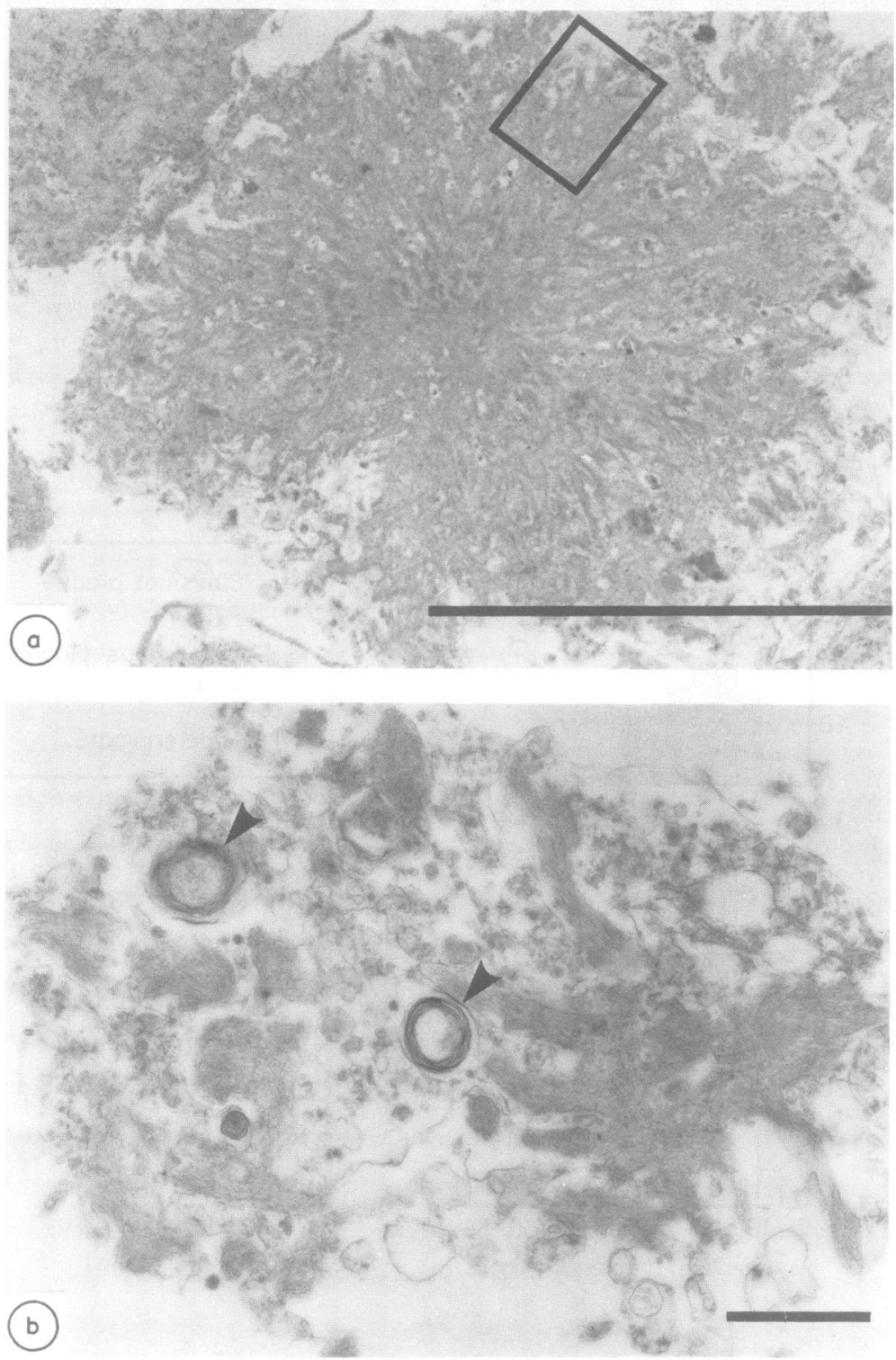

Fig 3 Isolated plaque cores from Alzheimer's disease brain SE139. (a) Typical appearance of a classical plaque core with diameter $>6 \mu \mathrm{m}$. Window indicates area shown in fig 6. Bar $=10 \mu \mathrm{m}$. (b) Core with diameter $<6 \mu \mathrm{m}$. Bar $=1 \mu \mathrm{m}$. Arrows = lamellated bodies. 


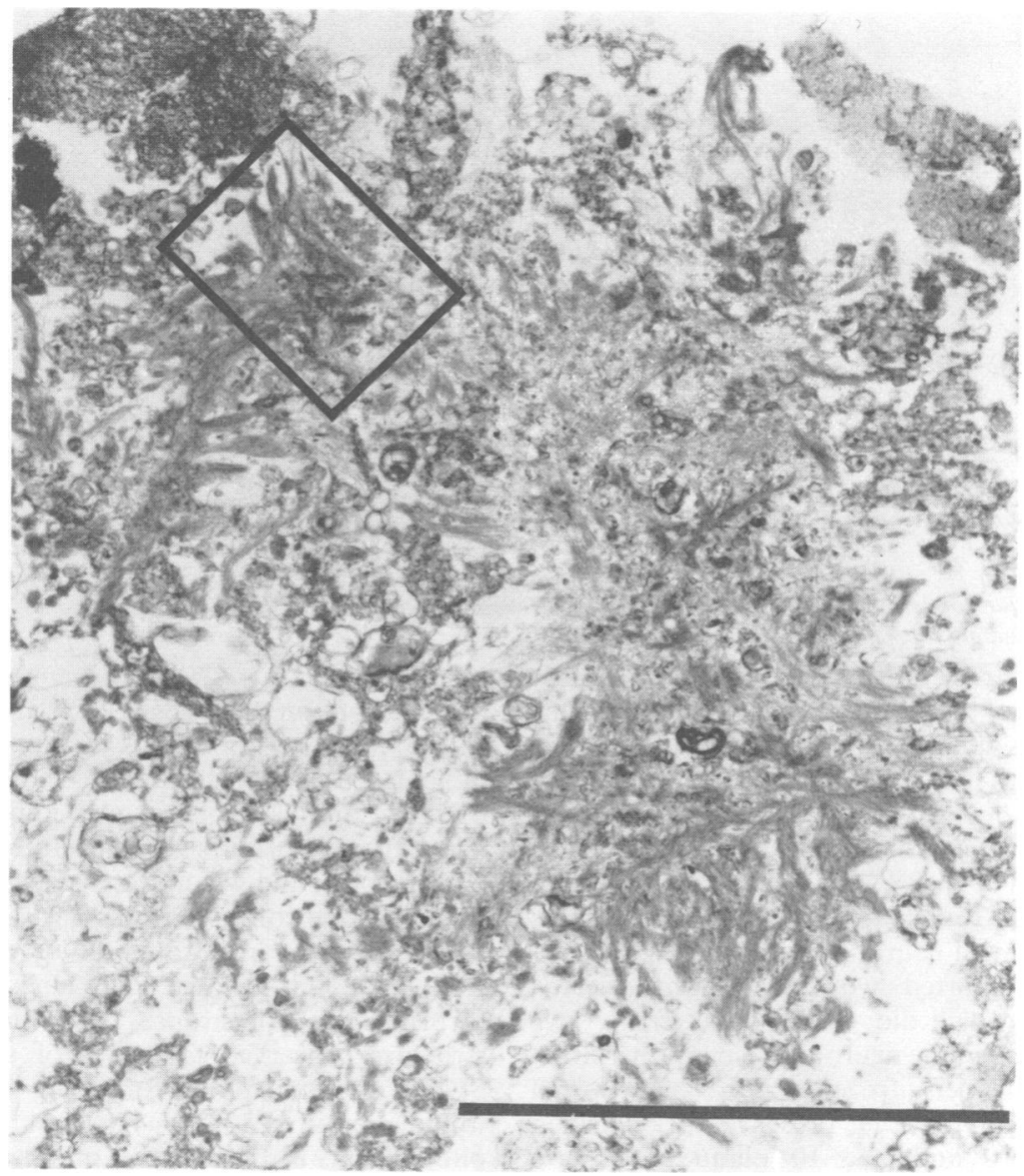

Fig 4 Large amorphous plaque core in the neuronal preparation from Down's syndrome brain M3. This type of structure has not been seen in the neuronal preparations from a number of Alzheimer brains prepared for electron microscopy. including SE139. Window indicates area shown in fig 7. Bar $=10 \mu \mathrm{m}$.

ined under a Philips 410 electron microscope. The data for fig $2 \mathrm{~b}$ were compiled from photographs taken during the systematic search of a large number of grids for plaque core profiles.

\section{Results}

\section{Light microscopical observations on isolated plaque} cores in neuronal suspensions

During the course of our studies on the biochemical nature of senile plaque amyloid ${ }^{19-21}$ we have produced neuronal preparations from a large number of post-mortem brains from patients with a diagnosis of Alzheimer's disease. These preparations consist mainly of neuronal cell bodies, naked nuclei, blood vessel fragments, and varying numbers of tanglebearing neuronal perikarya and isolated plaque cores.
Some brains yield neurofibrillary tangles in the absence of plaque cores. The preparation from SE139 contained both tangles and a large number of isolated plaque cores which had the classical appearance of a well-formed polarisation cross after Congo red staining, and a mean diameter of $16.0 \mu \mathrm{m} \pm 0.5$ (Standard error).

Two of the four Down's syndrome brains (M4 and M6) produced inadequate numbers of plaque cores. The other two (M3 and M5) produced normal plaque cores, but in both cases a high proportion (50 and $85 \%$ respectively) of plaque cores with an obviously different morphology were also observed (fig 1). These unusual "amorphous" plaque cores were less compact and more granular in appearance than classical plaque cores, and the polarisation crosses were poorly defined or more often non-existent. From the 


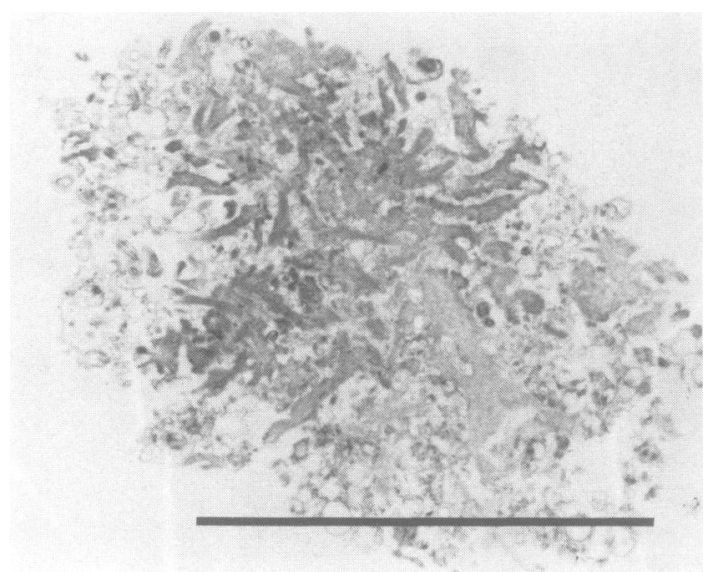

Fig 5 Isolated plaque core from Down's syndrome brain M3 with possibly intermediate morphology. This was considered as classical for the purposes of fig $2 \mathrm{~b}$. Bar $=10 \mu \mathrm{m}$.

size distributions of the classical and amorphous forms given in fig $2 \mathrm{a}$ it is apparent that the latter were, on the whole, larger, with a mean diameter of $29.6 \mu \mathrm{m} \pm 1.6$ for M3, $22 \cdot 8 \mu \mathrm{m} \pm 0.8$ for M5 compared with corresponding mean values for the classical plaque cores from these brains of $16.5 \pm 1.1 \mu \mathrm{m}$ and $12.7 \pm 1.0 \mu \mathrm{m}$ respectively. All four Down's syndrome brains showed neurons with neurofibrillary tangles.

In previous reported work on seven selected Alzheimer cases which did yield plaque cores, ${ }^{19}$ we found the numbers in the neuronal suspensions varied from $0.6-2.5 \times 10^{4}$ per $g$ frozen cortex. The neuronal preparations used for the present work yielded $7.3 \times 10^{4}, 1.4 \times 10^{4}$ and $0.3 \times 10^{4}$ plaque cores per $\mathrm{g}$ cortex for SE139, M3 and M5 respectively.

\section{Electron microscopical observations}

All of the sectioned plaque cores with an average diameter $>6 \mu \mathrm{m}$ from the Alzheimer's disease brain SE139 consisted of densely packed amyloid fibrils, often radially arranged to produce a circular outline (fig 3a); although more elongated forms, and also semicircular or crescentic shapes were seen.

Sections of SE139 plaque cores with a diameter $<6 \mu \mathrm{m}$ (presumably generated by grazing sections of larger structures) had lost this appearance (fig $3 \mathrm{~b}$ ). The amyloid in these sections was more randomly oriented, and amounted to a much lower proportion of the total plaque core material. In this respect these profiles were reminiscent of the much larger "amorphous" type of plaque core seen in Down's syndrome brain M3 which is to be described later.

Owing to the small number of plaque cores in the neuronal preparation from brain M5, and the resulting difficulty in finding a sufficient number: of plaque cores in sections, electron microscopical

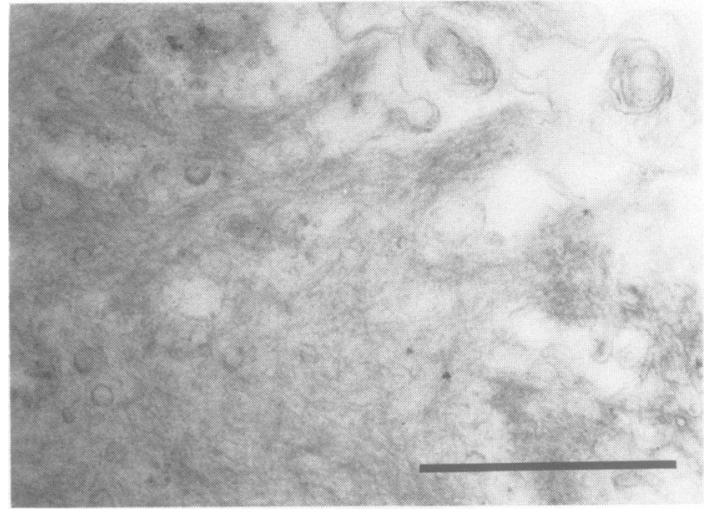

Fig 6 Part of the classical plaque core shown in fig $3 a$ at higher magnification showing the "feltwork" of intermeshing amyloid fibrils. Bar $=1 \mu \mathrm{m}$.

observations on the Down's cases are confined to M3. In M3, plaque core sections of diameter $<6 \mu \mathrm{m}$ were difficult to distinguish from those of similar size from SE139. For this reason it was impossible to decide which of these profiles were generated by the classicial plaque cores, and which were generated by the amorphous plaque cores seen by polarisation microscopy.

Sections of cores from M3 of diameter $>6 \mu \mathrm{m}$ were, in all but one case, easily divisible into classical forms whose appearance fell within the range of structures observed for cores of similar size from SE139; and amorphous forms (fig 4) which were not found in brain SE139. We have not found these amorphous forms in the electron microscopy of neuronal fractions from any other Alzheimer's disease brain. As expected, the classical and amorphous profiles were seen in approximately equal numbers (fig $2 \mathrm{~b}$ ). One plaque core section was observed in brain M3 which could be described as intermediate in morphology (fig 5); this was not sufficiently unusual to be considered as amorphous, and so was assessed as classical for fig $2 \mathrm{~b}$.

The amyloid in the large amorphous isolated plaque cores was usually present in the form of loosely packed bundles $0 \cdot 2-0.4 \mu \mathrm{m}$ across, which were not organised radially around a single centre (as in classical plaque cores), but were either acentric or multi-centric. The bundles themselves were very loosely arranged, and separated by unusually large amounts of the type of cell debris found by previous workers ${ }^{1213}$ to form an integral part of plaque ultrastructure (as described below). Regions of some of the amorphous plaque cores contained minimal amounts of amyloid which was sometimes present in the form of isolated single strands rather than in bundles. Differences in the ultrastructure of classical and 


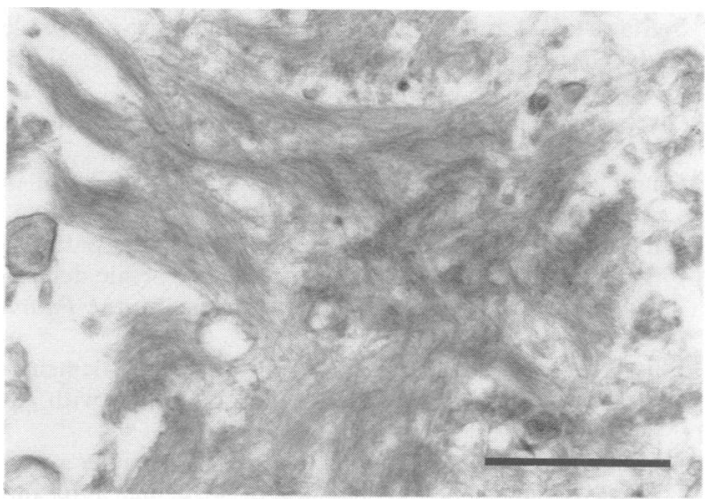

Fig 7 High power picture of part of an amorphous plaque core from Down's syndrome brain M3 (see fig 4) showing the bundles formed from loosely packed, parallel amyloid fibrils. Bar $=1 \mu \mathrm{m}$.

amorphous plaques were also demonstrable at high power. The amyloid in the classical plaques was seen to form a "feltwork" of interlacing fibrils the majority of which were radially oriented (fig 6). In contrast, the amyloid fibrils forming the bundles seen in the amorphous form were less tightly packed and usually ran a parallel course within each bundle; with the bundles themselves crossing over each other (fig 7).

Some of the degenerating material intervening between the bundles of filaments in the larger amorphous cores seemed to consist of cell membrane profiles (fig 8), some containing more or less normal organelles similar to those seen at the periphery of a classical plaque. However, the majority of this material consisted of lamellated bodies, degenerating mitochondria, vesicles and small myelin forms and therefore resembled more closely the type of debris found in much smaller amounts entangled with the amyloid of the classical plaque core.

\section{Discussion}

It is important to bear in mind that the above observations were made on plaque cores isolated from cerebral cortex, and not on intact plaques. The isolation method involves forcing finely chopped cortical tissue through stainless steel meshes of diminishing aperture; the smallest aperture being $74 \mu \mathrm{m}$. The resultant suspension is then centrifuged through a layer of $30 \%$ sucrose to produce a preparation (the neuronal fraction) containing large numbers of neuronal cell bodies with shorn off axons and dendrites, together with the isolated plaque cores. In this work the plaque cores were not further purified by the proteolytic digestion and sucrose density gradient centrifugation steps described previously. ${ }^{1920}$

The morphology of the plaque cores isolated by

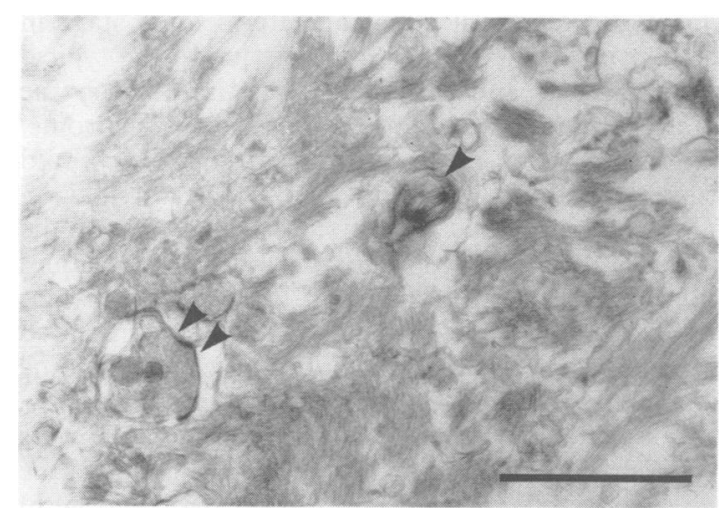

Fig 8 Part of an amorphous plaque at higher magnification. Single arrow = lamellated body. Double arrow = possibly degenerate cell process - this has some resemblance to a synaptosome. Bar $=1 \mu \mathrm{m}$.

this procedure must be correlated with the ultrastructural appearance of whole plaques in situ. According to Wisniewski and Terry ${ }^{14}$ the earliest stage in plaque genesis in Alzheimer's disease is the formation of a few abnormal neurites with no amyloid. Further neurite degeneration, along with the deposition of wisps of amyloid visible only at the level of the electron microscope, leads to the formation of the "primitive" plaque. The process of degeneration continues, and a central core of amyloid is formed giving the "typical" or "classical" plaque structure. Eventually, the neurites degenerate completely, leaving only a mass of amyloid, which constitutes the "compact" or "burned-out" plaque.

The isolated "classical" plaque cores (which are the only type we have found in neuronal preparations from the brains of cases of Alzheimer's disease) could be produced from both typical and burned-out plaques. In a typical plaque, the sharp boundary, which is often present between the amyloid core and the periphery, would allow shearing forces to separate the latter so that it is lost.

It is difficult, however, to envisage production of "amorphous" isolated plaque cores (which we observed in two Down's syndrome brains) from any of the three classes of plaque mentioned above. It seems likely, therefore, that the two Down's syndrome brains contained large numbers of a fourth type of plaque, which generates the "amorphous" plaque core structure upon disruption, and which has not been found by us so far in the brains of patients with Alzheimer's disease. The amorphous cores have a structure (a loose meshwork of amyloid filaments holding together a mass of partially degraded cellular material) that is reminiscent of the primitive plaque but with much more extensive deposits of amyloid. 
This could represent either an intermediate stage in the conversion of the primitive plaque to the typical plaque, or an alternative structure into which the primitive plaque may develop in Down's syndrome. The latter seems more likely as the former would require substantial rearrangement of the amyloid.

Whereas previous workers ${ }^{1-7}$ have stressed the similarity of the neuropathological changes (plaques and neurofibrillary tangles) in Alzheimer's disease and later Down's syndrome, the results presented here indicate that these two conditions can show some significant differences in plaque morphology. Although this conclusion is based on two Down's syndrome brains, Masters et al $^{15}$ have isolated plaque cores (by a different procedure from that used here) from a number of Alzheimer's disease brains and one Down's syndrome brain. These authors noted that in the case of Down's syndrome "morphologically, the amyloid cores... were predominantly of the amorphous type" whereas in Alzheimer's disease "the predominant form is the dense spherical core with a Maltese cross in polarised light".

Disparities in plaque morphology between Alzheimer's disease and Down's syndrome may be understood simply as a reflection of two distinct genotypes interacting differently in the process of plaque formation. It is tempting to speculate on the process which leads to the more diffuse mode of amyloid deposition in the amorphous plaques but the circumstances in which plaque cores are laid down are too uncertain for useful conclusions to be made. The most striking feature of these plaques is that they show no evidence of deposition of amyloid around a nucleating site, unlike the classical amyloid plaque. This difference is not due to a more rapid formation of plaques in Down's syndrome (if indeed plaque formation is faster in this condition), as this feature is not found with scrapie or Creutzfeldt Jakob disease plaques, which may likewise be formed more rapidly than in Alzheimer's disease.

In the light of these observations, a thorough comparative study of plaque morphology in situ of Alzheimer's disease and Down's syndrome is indicated for future work. If substantiated, differences in plaque morphology must be taken into account in the use of Down's syndrome as a model for Alzheimer's disease.

We thank the Wellcome Trust for financial support and Miss W Tomlinson for technical assistance.

\section{References}

${ }^{1}$ Malamud N. Neuropathology of organic brain syndromes associated with ageing. In: Gaitz CM, ed. Ageing and the Brain. New York: Plenum Press 1972:63-87.

${ }^{2}$ Wisniewski KE, Wisniewski HM, Wen GY. Occurrence of neuropathological changes and dementia of Alzheimer's disease in Down's syndrome. Ann Neurol 1985;17:278-82.
${ }^{3}$ Solitaire GB, Lamarche JB. Alzheimer's disease and senile dementia as seen in Mongoloids: neuropathological observations. Am J Ment Defic 1966;70:840-8.

${ }^{4}$ Olsen MI, Shaw G-M. Presenile dementia and Alzheimer's disease in Mongolism. Brain 1969;92:147-56.

${ }^{5}$ Ohara PT. Electron microscopical study of the brain in Down's syndrome. Brain 1972;95:681-4.

${ }^{6}$ Burger PG, Vogel FS. The development of the pathological changes of Alzheimer's disease and senile dementia in patients with Down's syndrome. Am J Pathol 1973;73:457.

${ }^{7}$ Ellis WG, McCulloch JR, Corley CL. Presenile dementia in Down's syndrome: Ultrastructural identity with Alzheimer's disease. Neurology 1974;24:101-6.

${ }^{8}$ Mann DMA, Yates PO, Marcyniuk B. Alzheimer's presenile dementia, senile dementia of Alzheimer type and Down's syndrome in middle age form an age related continuum of pathological changes. Neuropathol Appl Neurobiol 1984;10:185-207.

${ }^{9}$ Price DL, Whitehouse PJ, Struble RG, et al. Alzheimer's disease and Down's syndrome. Ann NY Acad Sci 1982;396:145-64.

${ }^{10}$ Yates CM, Simpson J, Maloney AFJ, Gordon A, Reid AH. Alzheimer-like cholinergic deficiency in Down's syndrome. Lancet 1980;ii:979.

${ }^{11}$ Kidd M. Paired helical filaments in electron microscopy of Alzheimer's disease. Nature 1963;97:192-93.

${ }^{12}$ Kidd M. Alzheimer's disease. An electron microscopical study. Brain 1964;87:307-20.

${ }^{13}$ Terry RD, Gonatas HK, Weiss M. Ultrastructural studies in Alzheimer's presentile dementia. Am J Pathol 1964;44:269-97.

${ }^{14}$ Wisniewski HM, Terry RD. Re-examination of the pathogenesis of the senile plaque. In: Zimmerman HM, ed. Progress in Neuropathology. New York and London: Grune and Stratton 1973;2:26.

15 Masters CL, Simms G, Weinmann NA, Multhaup G, McDonald BL, Bayreuther K. Amyloid plaque core protein in Alzheimer's disease and Down's syndrome. Proc Natl Acad Sci USA 1985;82:4245-9.

${ }^{16}$ Glenner GG, Wong CW. Alzheimer's disease: Initial report of the purification and characterisation of a novel cerebrovascular amyloid protein. Biochem Biophys Res Commun 1984;120:885-90.

${ }^{17}$ Glenner GG, Wong CW. Alzheimer's disease and Down's syndrome: Sharing of a unique cerebrovascular amyloid fibril protein. Biochem Biophys Res Commun 1984;122:1131-5.

${ }^{18}$ Kidd M, Allsop D, Landon M. Senile plaque amyloid, paired helical filaments and cerebrovascular amyloid in Alzheimer's disease are all deposits of the same protein. Lancet 1985;i:278.

${ }^{19}$ Allsop D, Landon M, Kidd M. The isolation and amino acid composition of senile plaque core protein. Brain Res 1983;259:348-52.

${ }^{20}$ Kidd M, Allsop D, Landon M. Senile plaque amyloid. In: Clifford Rose F. ed. Modern Approaches to the Dementias. Part 1: Etiology and Pathophysiology. Interdiscipl Topics Geront. Basel. Karger 1985;19:114-26.

${ }^{21}$ Allsop D, Landon M, Kidd M. Cerebral amyloid and Alzheimer's syndrome. In: Glenner GG and Osserman E eds. Proc VIth International Conference on Amyloidosis. New York. Plenum (in press). 